\title{
CONSORT-Equity 2017 extension and elaboration for better reporting of health equity in randomised trials
}

\author{
Vivian A Welch, ${ }^{1,2}$ Ole F Norheim, ${ }^{3,4}$ Janet Jull, ${ }^{5}$ Richard Cookson, ${ }^{6}$ Halvor Sommerfelt, ${ }^{3,7}$ \\ Peter Tugwell, ${ }^{8}$ CONSORT-Equity and Boston Equity Symposium
}

${ }^{1}$ Methods Centre, Bruyère

Research Institute, Ottawa, ON,

Canada.

${ }^{2}$ School of Epidemiology,

Public Health and Preventive

Medicine, University of Ottawa

${ }^{3}$ Centre for Intervention Science in Maternal and Child Health

(CISMAC), Department of Global

Public Health and Primary Care,

University of Bergen, Bergen,

Norway

${ }^{4}$ Department of Global Health and Population, Harvard TH Chan School of Public Health,

Bergen, Norway

${ }^{5}$ Ottawa Hospital Research

Institute, University of Ottawa

${ }^{6}$ Centre for Health Economics,

University of York, York Y010

5DD, UK

${ }^{7}$ Norwegian Institute of Public Health, Oslo, Norway

${ }^{8}$ Department of Medicine,

University of Ottawa

Correspondence to: VA Welch vwelch@campbellcollaboration. org

Additional material is published online only. To view please visit the journal online.

Cite this as: BMJ 2017;359:j5085 http://dx.doi.org/10.1136/bmj.j5085

Accepted: 4 October 2017
We outline CONSORT-Equity 2017

reporting standards, an extension to the CONSORT (Consolidated Standards of Reporting Trials) statement that aims to improve the reporting of intervention effects in randomised trials where health equity is relevant. Health inequities are unfair differences in health that can be avoided by reasonable action. We defined a randomised trial where health equity is relevant as one that assesses effects on health equity by evaluating an intervention focused on people experiencing social disadvantage or by exploring the difference in the effect of the intervention between two groups (or as a gradient across more than two groups) experiencing different levels of social disadvantage, or both. We held a consensus meeting with diverse potential users from high, middle, and low income countries, including knowledge users such as patients and methodologists. We discussed evidence for each proposed extension item from empirical studies, reviews, key informant interviews, and an online survey, aiming to improve clarity of

\section{SUMMARY POINTS}

- The CONSORT statement provides a minimum set of 25 items to be reported with rationale and exemplars for all randomised trials

- A multidisciplinary team used a consensus approach to develop CONSORTEquity 2017, an extended CONSORT reporting guideline for better design and reporting of randomised trials where equity is relevant

- CONSORT-Equity 2017 extends 16 items of the CONSORT statement and adds a new item on ethical concerns for transparently reporting information relevant to assessment of effects on health equity

- The use of the CONSORT-Equity 2017 guideline will improve reporting of health equity in randomised trials and thereby facilitate greater use of this information in decision making

reporting without imposing undue burden on authors. The new guidance contains equity extensions to 16 items from CONSORT 2010 plus one new item on research ethics reporting, with examples of good practice and a brief explanation and elaboration for each. Widespread uptake of this guidance for the reporting of trials where health equity is relevant will make it easier for decision makers to find and use evidence from randomised trials to reduce unfair inequalities in health.

\section{Introduction}

Health inequalities are pervasive both between and within countries. In the US, the gap in life expectancy at age 40 between the top and bottom centiles of pre-tax household income is more than 10 years for women and 15 years for men. ${ }^{1}$ Health inequalities such as these are labelled "inequities" or "disparities" when they are considered unfair. Different views exist regarding which health inequalities are unfair, and what should or can be done to reduce them, but here we follow Marmot's elaboration. ${ }^{2}$ In this view, a key criterion for assessing whether a health inequality is unfair is whether it is considered to be avoidable by "reasonable action." 2 Reducing unfair inequalities in health is a matter of social justice and sound planning for improved population health and development. To achieve this goal, we need better evidence on what creates, maintains, and reduces health inequities. ${ }^{3}$ We need improved collection, reporting, and communication of such evidence to facilitate its use in decision making. ${ }^{4-8}$

Randomised trials, when completely and transparently reported, provide robust evidence about the effects of programmes and interventions because they can be designed to create groups that are balanced on both known and unknown confounders. Randomised trials are not limited to devices, drugs, or procedures, but are also widely used to assess complex health system and policy interventions, ${ }^{9-11}$ as well as international development and public health programmes. ${ }^{12} 13$ Randomised trials provide the opportunity to assess effects of interventions on health equity.

Our group developed a definition of a randomised trial relevant to health equity as one that evaluates an 
intervention focused on people experiencing social disadvantage or that explores the different effects of an intervention between two or more groups experiencing different levels of social disadvantage, or both. ${ }^{14}$ Trials may meet both criteria if they are focused on a disadvantaged population and also assess subgroup differences. This definition does not depend on the stated trial objectives being related to health equitywe identified numerous trials that provided evidence about effects on health equity without having an explicit objective related to doing so.

We used the PROGRESS-Plus framework (box 1) to identify relevant factors that define population groups who may experience social disadvantage. ${ }^{16} 17$ These characteristics may be associated with each other (for example, educational attainment and socioeconomic status) and may interact (for example socioeconomic status and ethnicity), and their importance may depend on the context and setting. PROGRESS-Plus recognises more characteristics than PROGRESS alone, including personal characteristics, time-dependent situations and relationships. ${ }^{17}$

We included trials of non-medical interventions that are focused not directly on health but rather on social determinants of health, such as interventions that aim to reduce poverty. ${ }^{15}$ Although some types of trials were less likely to provide evidence about effects on health equity (such as small scale efficacy/explanatory trials), we found examples of these types of trials that did provide evidence across one or more PROGRESS characteristic.

Most reports of randomised trials include few details on the social and demographic characteristics of participants, ${ }^{18}{ }^{19}$ and some disadvantaged populations tend to be systematically underrepresented, such as women, older adults, and ethnic minorities. ${ }^{20}$ Furthermore, analyses to explore effect measure modification across individual or population level characteristics related to socioeconomic strata are rarely done; when they are done, they are rarely reliable owing to small numbers. ${ }^{21}$ This problem has been identified for different characteristics including sex/gender, ${ }^{22}$ advanced age, ${ }^{23}$ and literacy. ${ }^{24}$ The lack of such details makes judgments about the applicability of randomised trial results to these populations difficult. In cluster randomised trials, where the unit of randomisation is a whole clinic or community, hidden disadvantaged populations not be appropriately identified or described. A second problem is that in randomised trials that are focused on people who experience health inequities (such as those for decision aids for low literacy populations or social support for low income seniors), details on question formulation, context, and applicability to inform decisions about equitable policies and programmes are lacking. ${ }^{25}$

One way to improve reporting of randomised trials is the development, dissemination, endorsement, and implementation of reporting guidelines. Journal endorsement of the CONSORT statement 2010 (Consolidated Standards Of Reporting Trials) has been shown to improve reporting of some aspects of randomised trials. ${ }^{26}$ It is the most widely used guidance for reporting trials and provides a minimum set of 25 items to be reported, with rationale and exemplars. However, the CONSORT statement does not contain any items specific to health equity, either in the main statement or in any of the extensions for different designs, outcomes, and interventions (www.consortstatement.org).

We developed an extension to the CONSORT statement to improve reporting of randomised trials relevant to health equity. As with previous reporting guidelines, this guideline aims to balance the objective of improved evidence for decision making with the burden of reporting for authors and requires the goodwill of authors and editors in recognising their value.

One set of beneficiaries of better reporting of health equity in trials are systematic reviewers and other analysts responsible for synthesising and communicating evidence about equity issues to decision makers. Synthesised evidence on health equity would be of interest to decision makers who seek information about distribution of effects in the population and, ultimately, members of the public, especially those from disadvantaged communities who are likely to benefit the most from equity oriented evidence.

\section{Objectives}

To develop a guideline to improve the reporting of effects of interventions on health equity in randomised controlled trials, for trials that assess effect measure modifications and for trials that are focused on disadvantaged populations.

\section{Methods}

We followed the steps proposed for developing consensus based reporting guidelines by Moher et al. ${ }^{27}$ We published a protocol describing our six phased approach (fig 1) to building a multidisciplinary team and advisory group to inform the steps to establish need, obtain funding, review the literature, conduct an online survey to gather opinions of stakeholders, and hold a consensus meeting. ${ }^{14}$ In addition, we conducted key informant interviews with potential users of the guidelines to identify their views on the ways in which health equity could be reported in randomised trials (to be published elsewhere).

We used an integrated knowledge translation approach throughout guideline development, ${ }^{28}$ by assembling a multidisciplinary research team and advisory board with participation of knowledge users (including policy makers, patient and community representatives, practitioners, funders, and journal editors) and methodologists (such as library scientists, trialists, economists, epidemiologists, and statisticians) in the design and conduct of the research and planning. These participants were carefully chosen because of their interest and expertise in health equity and their expertise in conducting, participating 


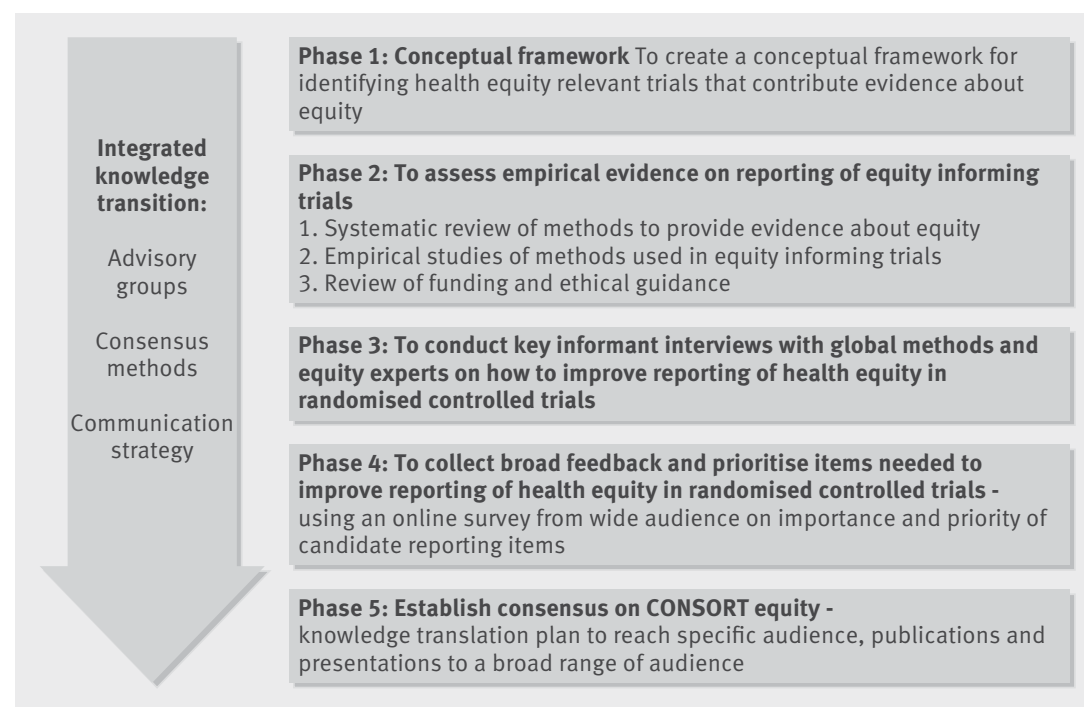

Fig 1 | Overall research plan as well as different stakeholders, including patients, policy makers, methodologists, and trialists (see web appendix 1 for survey distribution).

\section{Consensus meeting}

We held a two day meeting on health inequality in randomised controlled trials in Boston (22-23 September 2016), in collaboration with the Centre for Intervention Science in Maternal and Child Health. The number of participants was limited to $<30$ to enable discussion and engagement. Participants were chosen to be inclusive of different disciplinary and stakeholder perspectives.

The purpose of the meeting was to discuss the additional guidance needed beyond the CONSORT 2010 statement for health equity relevant trials, in the form of modified or additional items.

The meeting participants were provided with preworkshop material outlining the proposed items additional to those in the CONSORT statement, with data from empirical studies and other relevant guidance and policy statements (web appendix 2). The meeting included a debate on defining and measuring health inequalities and health equity and discussion of four working papers from experts involved in trials conducted by the Centre for Intervention Science in Maternal and Child Health (papers in preparation). After this discussion, we used an interactive approach to present results of the survey, empirical studies, related guidance, and the key informant study, as well as the rationale for each proposed item. The quantitative results of the survey were considered in addition to open ended qualitative comments from the survey, evidence from the methods studies, related guidance, and key informant interviews, and panel members' opinions to decide on whether to include each item. Each participant was invited to act as a rapporteur, presenter, or discussant for at least one item. The discussion was recorded, and minutes were taken. Final results were synthesised and agreed upon by all through email.

\section{Patient involvement}

We included one patient on our advisory board who contributed to all aspects of the study design through quarterly calls. We included two patients in our consensus meeting (AL, RG). These patients were involved in writing the manuscript and approving the final version and are included as authors.

\section{Online survey}

We developed an online survey to allow wide participation. This survey included the standard CONSORT items, proposed CONSORT-Equity extensions, and examples for each CONSORTEquity item (https://www.surveymonkey.com/r/ CONSORTEquity). Extension items were proposed based on an ongoing systematic review and methods study that reviews the standard CONSORT items and extensions (including the social and psychological interventions extension under development). ${ }^{14}$ The survey was sent to intended users; we aimed for disciplinary diversity across health and social sciences

\section{Results}

\section{Online survey}

We received 168 responses to the online survey (web table 1), with representation from knowledge users (clinicians $(21 \%)$, patients $(12 \%)$, policy makers (13\%), journal editors (18\%)) and methodologists (epidemiologists (29\%), trialists (20\%), and economists $(7 \%))$. We obtained $16 \%$ of responses from people based in low or middle income countries. Responses to the first round of the survey achieved greater than $80 \%$ agreement for each of 23 proposed additional items (across 16 of the standard CONSORT items, as some are multiple subsets of one item; for example, items $1 \mathrm{c}$ and $1 \mathrm{~d}$ are additional items that extend item 1) for trials relevant to health equity (web table 2). Therefore, we did not conduct a second round survey. In addition, these results of $>80 \%$ agreement were consistent for different types of respondents, including people from low and middle income 
countries $(n=32)$, patients $(n=15)$, trialists $(n=59)$, researchers $(n=90)$, journal editors $(n=22)$, policy makers $(n=16)$, and practitioners $(n=26)$. Survey participants provided detailed comments to support their ratings, which were presented with the survey results to foster discussion about the proposed items at the Boston Equity Symposium.

\section{Consensus meeting}

The Boston Equity Symposium participants and the CONSORT-Equity team (web table 3), (hereafter referred to as "we") reached agreement on extensions for 16 items of the standard CONSORT statement and one new item on ethical concerns (with two subitems). The criteria for inclusion was agreement by consensus voting of all participants at the Boston Equity Symposium. For example, one item supported by respondents to the online survey was removed because the panel members decided that it was too similar to another agreed extension item. In the consensus meeting, we agreed to focus only on extension items needed for trials relevant to health equity, not changes to the main CONSORT statement. We also agreed that the reporting items should focus on transparency of what was done and how, rather than providing guidance on which methods should be used. Finally, we decided to focus this reporting guideline on randomised trials, although some of the items may also be useful in reporting other study designs, such as non-randomised studies and population modelling studies.

\section{Extension of CONSORT 2010 to health equity relevant trials}

The original CONSORT statement (25 items) and CONSORT-Equity extension items are shown in table 1. We developed extensions for 16 items of the CONSORT 2010 statement, and we agreed on one new item on ethical concerns. This paper is a combination of a reporting guideline and an explanation and elaboration document.

\section{Title and abstract}

Item $1 \mathrm{a}$

Standard CONSORT item-Identification as a randomised trial in the title.

Extension for health equity relevant trials-If health equity is a major focus, consider using the term "health equity" in the title.

Example 1 (using equity)_-“Impact of a peercounseling intervention on breastfeeding practices in different socioeconomic strata: results from the equity analysis of the PROMISE-EBF trial in Uganda."29

Example 2 (using specific descriptors)_-"Impact of maternal education about complementary feeding on their infants' nutritional outcomes in low- and middleincome households: a community-based randomised interventional study in Karachi, Pakistan.”30

Explanation-The title is an opportunity to help patients (or the public), policy makers, and practitioners use of health equity relevant trials by making them easier to find. However, we decided that it was not reasonable to insist on use of the term "health equity" because health equity is multidimensional and might be better described by more specific terms. For example, we identified over 30 examples of health equity relevant randomised trials that used terms such as "remote," "homeless," or "low income" in the title. We agreed that these terms could be more informative than the term "health equity" by allowing a user interested in a specific dimension, such as socioeconomic status, to identify relevant trials. We propose considering the term "health equity" in the title, if relevant. In addition, to improve ease of searching, we propose that trial authors and journals use the $2016 \mathrm{MeSH}$ term "health equity" for indexing.

\section{Item $1 b$}

Standard CONSORT item-Structured summary of trial design, methods, results, and conclusions (for specific guidance see CONSORT for abstracts)

Extension for health equity relevant trials-State research question(s) related to health equity.

Example-"To examine educational gradients in dementia care and whether the effect of a dementia collaborative care management intervention varied according to the educational attainment of the informal caregiver." 31

Explanation-We endorsed the need for reporting health equity objectives in the abstract for the same reason; namely to enhance ease of finding them for policy-makers, practitioners and patients.

\section{Item $1 \mathrm{C}$}

Standard CONSORT item-None.

Extension for health equity relevant trials-Present results of all planned health equity analyses.

Example-"Results did not differ substantially when stratified by age, sex, race/ethnicity, or neighborhood poverty rate."32

Explanation-Results of planned health equity analyses should be described in the abstract, regardless of the size, direction, or statistical significance, to facilitate their interpretation by policy makers, practitioners, and patients for decisions.

Item 1d

Standard CONSORT item-None.

Extension for health equity relevant trials-Describe extent and limits of applicability to populations of interest across PROGRESS-Plus characteristics.

Example-"For relatively food-secure populations, educational interventions about appropriate CF [complementary feeding] to mothers had a direct positive impact on linear growth of their infants." 30

Explanation-Health equity relevant trials may focus on specific populations defined across one or multiple axes of PROGRESS-Plus and may have limited applicability to other disadvantaged populations in different settings with different characteristics. These considerations should be described. 


\begin{tabular}{|c|c|c|c|}
\hline $\begin{array}{l}\text { Section/topic } \\
\text { and item No }\end{array}$ & Standard CONSORT item & Proposed extension for equity & Page No \\
\hline \multicolumn{4}{|c|}{ Title and abstract } \\
\hline $1 \mathrm{a}$ & Identification as a randomised trial in the title & $\begin{array}{l}\text { If health equity is a major focus, consider using the term "health } \\
\text { equity" in the title. }\end{array}$ & \\
\hline $1 b$ & $\begin{array}{l}\text { Structured summary of trial design, methods, results, and conclu- } \\
\text { sions (for specific guidance see CONSORT for abstracts) }\end{array}$ & State research question(s) related to health equity & \\
\hline $1 \mathrm{C}$ & & Present results of all planned health equity analyses & \\
\hline $1 d$ & & $\begin{array}{l}\text { Describe extent and limits of applicability to populations of interest } \\
\text { across PROGRESS-Plus characteristics }\end{array}$ & \\
\hline \multicolumn{4}{|c|}{ (6) } \\
\hline \multicolumn{4}{|c|}{ Background and objectives } \\
\hline $2 \mathrm{a}$ & Scientific background and explanation of rationale & Describe rationale for focus on health equity & \\
\hline $2 \mathrm{~b}$ & Specific objectives or hypotheses & State the objective being addressed with reference to health equity & \\
\hline \multicolumn{4}{|c|}{ ( } \\
\hline \multicolumn{4}{|l|}{ Trial design: } \\
\hline $3 a$ & $\begin{array}{l}\text { Description of trial design (such as parallel, factorial) including } \\
\text { allocation ratio }\end{array}$ & $\begin{array}{l}\text { Describe aspects of trial design that were chosen to answer equity } \\
\text { questions }\end{array}$ & \\
\hline $3 b$ & $\begin{array}{l}\text { Important changes to methods after trial commencement (such as } \\
\text { eligibility criteria), with reasons }\end{array}$ & & \\
\hline \multicolumn{4}{|l|}{ Participants: } \\
\hline $4 a$ & Eligibility criteria for participants & $\begin{array}{l}\text { Report population eligibility criteria across relevant PROGRESS-Plus } \\
\text { characteristics. }\end{array}$ & \\
\hline $4 b$ & Settings and locations where the data were collected & Report context and relationship to health inequity & \\
\hline $4 C$ & & $\begin{array}{l}\text { Report details of partnerships with populations and communities, } \\
\text { where applicable. }\end{array}$ & \\
\hline \multicolumn{4}{|l|}{ Intervention: } \\
\hline 5 & $\begin{array}{l}\text { The interventions for each group with sufficient details to allow rep- } \\
\text { lication, including how and when they were actually administered }\end{array}$ & $\begin{array}{l}\text { Report whether comparator intervention is the standard of care, } \\
\text { and whether it has equity implications. }\end{array}$ & \\
\hline \multicolumn{4}{|l|}{ Outcomes: } \\
\hline $6 a$ & $\begin{array}{l}\text { Completely defined pre-specified primary and secondary outcome } \\
\text { measures, including how and when they were assessed }\end{array}$ & $\begin{array}{l}\text { Report whether outcomes were identified as relevant and important } \\
\text { to population(s) across PROGRESS-Plus characteristics and how } \\
\text { this was done }\end{array}$ & \\
\hline $6 b$ & Any changes to trial outcomes after the trial commenced, with reasons & & \\
\hline \multicolumn{4}{|l|}{ Sample size: } \\
\hline $7 a$ & How sample size was determined & $\begin{array}{l}\text { Report whether analyses focused on health equity objectives are } \\
\text { powered to detect differences. }\end{array}$ & \\
\hline $7 b$ & $\begin{array}{l}\text { When applicable, explanation of any interim analyses and stopping } \\
\text { guidelines }\end{array}$ & & \\
\hline \multicolumn{4}{|l|}{ Randomisation } \\
\hline \multicolumn{4}{|c|}{ Sequence generation: } \\
\hline $8 \mathrm{a}$ & Method used to generate the random allocation sequence & & \\
\hline $8 b$ & $\begin{array}{l}\text { Type of randomisation; details of any restriction (such as blocking } \\
\text { and block size) }\end{array}$ & $\begin{array}{l}\text { Report whether randomisation was stratified on PROGRESS-Plus } \\
\text { characteristic(s) }\end{array}$ & \\
\hline \multicolumn{4}{|c|}{ Allocation concealment mechanism: } \\
\hline 9 & $\begin{array}{l}\text { Mechanism used to implement the random allocation sequence } \\
\text { (such as sequentially numbered containers), describing any steps } \\
\text { taken to conceal the sequence until interventions were assigned }\end{array}$ & & \\
\hline \multicolumn{4}{|l|}{ Implementation: } \\
\hline 10 & $\begin{array}{l}\text { Who generated the random allocation sequence, who enrolled } \\
\text { participants, and who assigned participants to interventions }\end{array}$ & & \\
\hline \multicolumn{4}{|l|}{ Blinding: } \\
\hline $11 \mathrm{a}$ & $\begin{array}{l}\text { If done, who was blinded after assignment to interventions (for } \\
\text { example, participants, care providers, those assessing outcomes) } \\
\text { and how }\end{array}$ & & \\
\hline $11 \mathrm{~b}$ & If relevant, description of the similarity of interventions & & \\
\hline \multicolumn{4}{|c|}{ Statistical methods: } \\
\hline $12 \mathrm{a}$ & $\begin{array}{l}\text { Statistical methods used to compare groups for primary and } \\
\text { secondary outcomes }\end{array}$ & & \\
\hline $12 b$ & $\begin{array}{l}\text { Methods for additional analyses, such as subgroup analyses and } \\
\text { adjusted analyses }\end{array}$ & $\begin{array}{l}\text { Report details of additional analyses focused on health equity, } \\
\text { including whether analyses to estimate heterogeneity of effects } \\
\text { between population subgroups were done on an additive or multi- } \\
\text { plicative scale, and whether pre-specified. }\end{array}$ & \\
\hline \multicolumn{4}{|c|}{ Ethical concerns } \\
\hline New item & & Report details of ethical clearance and informed consent & \\
\hline \multicolumn{4}{|l|}{ Results } \\
\hline \multicolumn{4}{|c|}{ Participant flow (a diagram is strongly recommended): } \\
\hline $13 a$ & $\begin{array}{l}\text { For each group, the numbers of participants who were randomly } \\
\text { assigned, received intended treatment, and were analysed for the } \\
\text { primary outcome }\end{array}$ & $\begin{array}{l}\text { Describe for each group, numbers of participants who were } \\
\text { assigned, received and who were analysed across relevant PRO- } \\
\text { GRESS-Plus characteristics }\end{array}$ & \\
\hline
\end{tabular}




\begin{tabular}{|c|c|c|c|}
\hline $\begin{array}{l}\text { Section/topic } \\
\text { and item No }\end{array}$ & Standard CONSORT item & Proposed extension for equity & Page No \\
\hline $13 b$ & $\begin{array}{l}\text { For each group, losses and exclusions after randomisation, together } \\
\text { with reasons }\end{array}$ & $\begin{array}{l}\text { Describe for each group, losses and exclusions after randomisation } \\
\text { across relevant PROGRESS-Plus characteristics, with reasons. }\end{array}$ & \\
\hline \multicolumn{4}{|l|}{ Recruitment: } \\
\hline $14 \mathrm{a}$ & Dates defining the periods of recruitment and follow-up & $\begin{array}{l}\text { Report whether methods of recruitment were designed to reach } \\
\text { populations across relevant PROGRESS-Plus characteristics. }\end{array}$ & \\
\hline $14 \mathrm{~b}$ & Why the trial ended or was stopped & & \\
\hline \multicolumn{4}{|l|}{ Baseline data: } \\
\hline 15 & $\begin{array}{l}\text { A table showing baseline demographic and clinical characteristics } \\
\text { for each group }\end{array}$ & $\begin{array}{l}\text { Present the baseline characteristics also across relevant PRO- } \\
\text { GRESS-Plus characteristics. }\end{array}$ & \\
\hline \multicolumn{4}{|c|}{ Numbers analysed: } \\
\hline 16 & $\begin{array}{l}\text { For each group, number of participants (denominator) included in } \\
\text { each analysis and whether the analysis was by original assigned } \\
\text { groups }\end{array}$ & & \\
\hline \multicolumn{4}{|c|}{ Outcomes and estimation: } \\
\hline $17 a$ & $\begin{array}{l}\text { For each primary and secondary outcome, results for each group, } \\
\text { and the estimated effect size and its precision (such as } 95 \% \\
\text { confidence interval) }\end{array}$ & & \\
\hline $17 b$ & $\begin{array}{l}\text { For binary outcomes, presentation of both absolute and relative } \\
\text { effect sizes is recommended }\end{array}$ & & \\
\hline \multicolumn{4}{|c|}{ Ancillary analysis: } \\
\hline $18 a$ & $\begin{array}{l}\text { Results of any other analyses performed, including subgroup analyses } \\
\text { and adjusted analyses, distinguishing pre-specified from exploratory }\end{array}$ & $\begin{array}{l}\text { Give the results of additional analytic approaches related to equity } \\
\text { objectives distinguishing pre-specified from exploratory. }\end{array}$ & \\
\hline $18 b$ & & $\begin{array}{l}\text { Details of implementation (coverage, intensity) in each trial arm } \\
\text { across relevant PROGRESS-Plus characteristics }\end{array}$ & \\
\hline \multicolumn{4}{|c|}{ 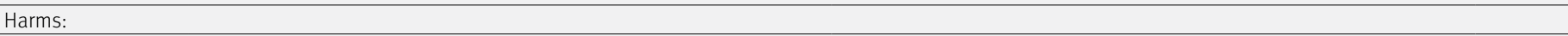 } \\
\hline 19 & $\begin{array}{l}\text { All important harms or unintended effects in each group (for specif- } \\
\text { ic guidance see CONSORT for harms) }\end{array}$ & $\begin{array}{l}\text { Report whether intervention generated inequities (e.g. unintended } \\
\text { effects) were assessed }\end{array}$ & \\
\hline \multicolumn{4}{|l|}{ Discussion } \\
\hline \multicolumn{4}{|l|}{ Limitations: } \\
\hline 20 & $\begin{array}{l}\text { Trial limitations, addressing sources of potential bias, imprecision, } \\
\text { and, if relevant, multiplicity of analyses }\end{array}$ & Report any limitations related to assessing effects on health equity. & \\
\hline \multicolumn{4}{|l|}{ Generalisability: } \\
\hline 21 & Generalisability (external validity, applicability) of the trial findings & $\begin{array}{l}\text { In addition, report applicability related to population of interest } \\
\text { across PROGRESS-Plus characteristics. }\end{array}$ & \\
\hline \multicolumn{4}{|l|}{ Interpretation: } \\
\hline 22 & $\begin{array}{l}\text { Interpretation consistent with results, balancing benefits and } \\
\text { harms, and considering other relevant evidence }\end{array}$ & & \\
\hline \multicolumn{4}{|c|}{ Other information } \\
\hline \multicolumn{4}{|c|}{ Registration: } \\
\hline 23 & Registration number and name of trial registry & & \\
\hline \multicolumn{4}{|l|}{ Protocol: } \\
\hline 24 & Where the full trial protocol can be accessed, if available & & \\
\hline \multicolumn{4}{|l|}{ Funding: } \\
\hline 25 & $\begin{array}{l}\text { Sources of funding and other support (such as supply of drugs), } \\
\text { role of funders }\end{array}$ & & \\
\hline
\end{tabular}

Introduction

Item $2 a$

Standard CONSORT item: Scientific background and explanation of rationale.

Extension for health equity relevant trials-Describe rationale for focus on health equity.

Example-"The present study targeted a streetbased population of African-American female sex workers who were disconnected from health services, and who also had very high levels of competing needs. As such, we hypothesized that the addition of a peer case manager would increase the efficaciousness of the [strengths-based case management] intervention for this high risk group of women."33

Explanation-For trials that are focused on answering a question relevant to health equity, the background must provide sufficient detail about why health equity is important in this setting for this intervention, supported by previous evidence, theory, or both.
Item $2 b$

Standard CONSORT item-Specific objectives or hypotheses.

Extension for health equity relevant trials: State the objective being addressed with reference to health equity.

Example (differential effects)-“The aim of this paper was to conduct a pre-specified subgroup analysis of the eGAME study to examine the consistency of trial effects by ethnicity, sex and baseline cardiovascular fitness." 34

Explanation-If the trial planned to assess differential effects, this should be stated as an objective.

\section{Methods}

\section{Item $3 a$}

Standard CONSORT item-Description of trial design (such as parallel, factorial) including allocation ratio. 
Extension for health equity relevant trials-Describe aspects of trial design that were chosen to answer equity questions.

Example 1-“Through collaboration, we were able to integrate our partner community priorities and requests into project design and analysis in meaningful ways. Community partners requested that the intervention reach all participants, not just an experimental group; we implemented a wait-listed randomised design.”35

Explanation-We propose explicit reporting of all aspects of study design that were chosen to answer equity questions. Clarity in reporting these elements of study design and analysis are helpful for systematic reviews.

Item $4 a$

Standard CONSORT item-Eligibility criteria for participants.

Extension for health equity relevant trialsReport population eligibility criteria across relevant PROGRESS-Plus characteristics.

Example-“. . . self-identification as black race and resides 2.5 hours or less driving time from the transplant center. Patients were excluded if they did not speak English.”36

Explanation-Population eligibility across PROGRESS-Plus (box 1) help to understand who was included (and who was not), which has implications for the applicability of findings; for example, exclusion of people beyond a certain driving distance or with certain language ability may affect applicability.

Item $4 \mathrm{~b}$

Standard CONSORT item-Settings and locations where the data were collected.

Extension for health equity relevant trials-Report context and relationship to health inequity.

Example-"Rural Thane district was selected to be the study setting due to its prevalence of high and early fertility, low contraceptive use, high unmet need for family planning, and limited access to government health services; the area is also characterized by high representation of tribal communities." 37

Explanation-The context of the study is essential for understanding the setting and relation to health inequity, as well as applicability.

Item $4 \mathrm{C}$

Standard CONSORT item-None.

Extension for health equity relevant trialsReport details of partnerships with populations and communities, where applicable.

Example-“Additionally, the chiefs of each village gave their approval for the study to take place in their village. Study participants provided verbal consent at the time of the surveys and coupons for the free ITNs [insecticide treated bed nets] were provided to all households in the study villages irrespective of whether or not they consented to participate in the study."38

Explanation-Authors should provide details about how communities were approached, informed, and engaged not only in the planning and conduct of research but also, as appropriate, in dissemination of the research and knowledge translation.

\section{Item 5}

Standard CONSORT item-The interventions for each group with sufficient details to allow replication, including how and when they were actually administered.

Extension for health equity relevant trials-Report whether the comparator intervention is the standard of care and whether it has equity implications.

Example-In the following example, the trial provided details on the accepted best standard of care. "WHO recommends that patients who screen negative for tuberculosis be placed on isoniazid preventive therapy ... . We designed the REMEMBER (reducing early mortality and early morbidity by empirical tuberculosis treatment regimens) study to assess whether empirical tuberculosis treatment will reduce early mortality compared with isoniazid preventive therapy in participants with advanced HIV disease presenting for antiretroviral therapy initiation in settings where tuberculosis is common." 39

Explanation-The health equity profile of a new intervention may be different depending on whether it is compared to the "best proven" standard of care, a less effective current standard of care, or an even less effective, outdated standard of care. ${ }^{40}$ The Helsinki Declaration (2013) recommends that a new intervention be compared to the "best proven" alternative except in situations where methodological reasons prevent this. ${ }^{41}$ In health equity relevant trials, clear reporting of the rationale for choice of comparator is important because it may be easier to justify a "second best" comparator as the current standard of care for vulnerable populations in resource poor settings. For example, a study in Thailand in 1999 compared short course zidovudine to placebo, even though long course zidovudine had been proven effective in reducing HIV-1 transmission by two thirds five years earlier in $1994 .^{42}$ However, the rationale for using placebo rather than long course as the comparator was not provided. Equity implications may also arise if some potential comparators are more expensive than others, and thereby divert resources

\section{Box 1: PROGRESS-Plus}

PROGRESS is used to define socially stratifying factors; it stands for place of residence, race/ethnicity/culture/religion/language, occupation, gender/sex, religion, education, socioeconomic status, and social capital. ${ }^{16}$ PROGRESS-Plus recognises additional characteristics including personal characteristics such as age or disability, features of relationships between people and their settings or other people (for example, being excluded from school) and time dependent relationships (for example, migrant status or discharge from hospital). ${ }^{17}$ Whether a characteristic represents disadvantage depends on social and cultural context, which may change over time; thus, considering context is particularly important. 
from other effective interventions. This could affect various social groups in different ways.

On the other hand, the comparator should be relevant to policy decisions in the given setting. Evidence on relative effectiveness and cost effectiveness of the intervention compared with the "best proven" alternative may produce results of less relevance if the current standard of care in a given setting is the "second best" treatment, such as a generic off-patent drug for low income settings. Comparing a new intervention with a "best proven" alternative may result in a high incremental cost effectiveness ratio, and the intervention may not be adopted for this reason. If "best proven" rather than actual standard of care is selected as the comparator, deprived populations may fail to get access to better care.

Item 6a

Standard CONSORT item-Completely defined prespecified primary and secondary outcome measures, including how and when they were assessed.

Extension for health equity relevant trials-Report whether outcomes were identified as relevant and important to population(s) across PROGRESS-Plus characteristics and how this was done.

Example-"For the present analyses, we used demographic characteristic measures, HIV/STI risk knowledge measures, and items on reported sexual behavior. All items were reviewed by community members, and modifications were made to the wording of some items to align them with the local vernacular." 35

Explanation-The patients on our consensus panel emphasised that the selection of relevant outcomes depends on the perspective of people affected by the problem. Thus, for health equity relevant trials, it is important to report how outcomes were selected, and whether these are outcomes that matter to socially disadvantaged people. Trials should report any serious discrepancies between the reported outcomes and those of interest to disadvantaged populations, and any efforts made to reconcile the two.

Item $7 a$

Standard CONSORT item-How sample size was determined.

Extension for health equity relevant trials-Report whether analyses focused on health equity objectives are powered to detect differences.

Example-"Due to funding restraints our maximum sample was fixed to 40 schools and up to 24 young people per school (see protocol for further details, [4]). We conducted a Monte Carlo power analysis to evaluate the power to detect moderation effects given our target and anticipated sample size (see Power Analysis in Supplement). The results suggested that there was good power to detect a moderation effect equivalent to a standardised regression coefficient of $>0.20$ but a lack of significant moderation would not be strong evidence against the presence of moderation effects smaller than this." ${ }^{43}$
Explanation-We propose that authors report if the study was powered to detect effects in a subgroup (such as women or people with low income) and whether it was powered to identify effect measure modification (that is, heterogeneity of effects between subgroups). For example, a cluster randomised trial in Malawi was designed with sufficient power to assess the differential effects of conditional cash transfers on girls in school and girls out of school at baseline. ${ }^{44}$

\section{Item $8 b$}

Standard CONSORT item-Type of randomisation; details of any restriction (such as blocking and block size).

Extension for health equity relevant trials-Report whether randomisation was stratified on PROGRESSPlus characteristics.

Example 1-"The randomisation was stratified by geographical region (Yorkshire, the North West, the South West Peninsula, and London and the South East) and quality of care (defined as being on and above, or below, the median on the key 12 indicator score)." ${ }^{45}$

Example 2-"Participants were randomly assigned following baseline testing via computer generated randomisation list (www.randomisation.com), stratified by gender in blocks of $4 . "{ }^{46}$

Explanation-Reporting details of stratification across characteristics used in analysis facilitates appraisal of the credibility of these analyses and with subgroup analyses in systematic reviews.

There are no extensions for the CONSORT items 9-11.

\section{Item $12 b$}

Standard CONSORT item-Methods for additional analyses, such as subgroup analyses and adjusted analyses.

Extension for health equity relevant trials-Report details of additional analyses focused on health equity, including whether analyses to estimate heterogeneity of effects between population subgroups were done on an additive or multiplicative scale, and whether prespecified.

Example - "Data were analysed by logistic regression with conservative variance estimates to take account of randomisation by hub-day clusters, including interaction tests for heterogeneity of effect by IMD [index of multiple deprivation] quintile ... In formal testing for heterogeneity of effects of age, sex, hub, and screening episode type by socioeconomic status, we used the continuous IMD score to increase statistical power." ${ }^{47}$

Explanation-Additional analyses may be helpful in assessing effects on health equity, which could include statistical approaches, such as tests of interaction, qualitative methods, or graphical approaches, such as directed acyclic graphs. ${ }^{48}$ The trial should report whether additional analyses focused on health equity were specified a priori, with rationale, and whether analyses to estimate heterogeneity of effects between population subgroups (that is, equity relevant effect measure modification) were done on an additive or multiplicative scale or both. 
New item: Ethical concerns

Standard CONSORT item-None.

Extension for health equity relevant trials-Report details of ethical clearance and informed consent.

Example-"Ethical clearance for the study was provided by the Boston University Medical Campus Institutional Review Board. Additional administrative approval was provided by the mayor of the town of Ambalavao, responsible for the villages in the district, and the Medicin Inspecteur of the Ambalavao health district, the local official in charge of all health related activities in the district." 38

Explanation-In the standard CONSORT 2010 statement, ${ }^{49}$ and previous extensions, ethics has been placed outside of the reporting guidelines because it is considered a journal responsibility, as described by the Council of Publishing Ethics. We have included ethical clearance and informed consent in this reporting guideline because socially disadvantaged populations may be particularly vulnerable in research and may need additional ethical protection. ${ }^{50-52}$

Ethical issues were an integral part of this multiphase project, with five ethicists involved (CW, ON, SE, KB, $\mathrm{GW}$ ). We reviewed national and international ethical guidance regarding the inclusion and protection of socially disadvantaged people. ${ }^{50-54}$ Ethical issues were emphasised in key informant interviews and in our face-to-face consensus meeting. In some cases, socially disadvantaged people (such as low income people or ethnic minorities) have been excluded inappropriately from research; in others, they have been over-represented because of convenience or willingness to participate (for example, for relatively little monetary compensation). ${ }^{50}$ Also, there are examples where whole communities or societies have been enrolled in research even though they were not likely to benefit from the findings. Reporting details of ethical approval and consent will allow the assessment of whether there was a fair distribution of burden and benefits for socially disadvantaged populations.

Item $13 a$

Standard CONSORT item-For each group, the numbers of participants who were randomly assigned, received intended treatment, and were analysed for the primary outcome.

Extension for health equity relevant trials-For each group, describe the numbers of participants who were assigned, received, and who were analysed across relevant PROGRESS-Plus characteristics.

Example-see fig 2 for a generic flowchart, adapted from the standard CONSORT flowchart. For example, a trial in New Zealand documented the number of Maori and non-Maori people who were randomised, discontinued, and completed the trial. ${ }^{55}$

Explanation-Describing participant flow according to PROGRESS-Plus enables analysis in relation to equity issues. Our consensus group expressed concerns that the flow diagram could become unwieldy if the factor of interest such as education (primary, secondary, postsecondary) had more than two levels or there was more than one characteristic of interest, such as poverty and rurality. In these cases, a disaggregated flow diagram might not be possible and other methods such as tables might be needed.

\section{Item $13 b$}

Standard CONSORT item-For each group, losses and exclusions after randomisation, together with reasons.

Extension for health equity relevant trials-For each group, describe losses and exclusions after randomisation across relevant PROGRESS-Plus characteristics, with reasons.

Example: see fig 2.

Explanation-As for participant flow, losses and exclusions across the PROGRESS-Plus factor of interest are important for trials that aim to assess heterogeneity of effects.

Item $14 a$

Standard CONSORT item-Dates defining the periods of recruitment and follow-up.

Extension for health equity relevant trials-Report whether methods of recruitment were designed to reach populations across relevant PROGRESS-Plus characteristics.

Example-"Participants were recruited from a sixth form college (where students study academic subjects) and a vocational college (where students learn work based skills) situated in the most deprived national quintile and identified as enrolling a high proportion of young people from deprived areas of a city in northern England."56

Explanation-Reporting whether recruitment was designed to reach populations across PROGRESS-Plus characteristics is important because this may affect generalisability. Recruitment may also deliberately exclude marginalised groups, such as those who intend to move out of the study area (or are at high risk of moving). The potential effect of exclusion criteria on recruitment of marginalised groups should be explicitly stated.

\section{Item 15}

Standard CONSORT item-A table showing baseline demographic and clinical characteristics for each group.

Extension for health equity relevant trials-Present the baseline characteristics across relevant PROGRESSPlus characteristics.

Example-One trial presented baseline characteristics (age, weight, education) for the total sample for each intervention group, as well as by sex for each intervention group (see web table 4). ${ }^{57}$

Explanation-If the trial has equity objectives, baseline characteristics should be reported across the PROGRESS-Plus characteristic of interest to display any baseline imbalances and allow the reader to assess the possible importance of adjusted analyses. Baseline clinical characteristics that are identical, or closely related, to trial outcomes (such as weight, school attendance, or health related behaviours) should be 


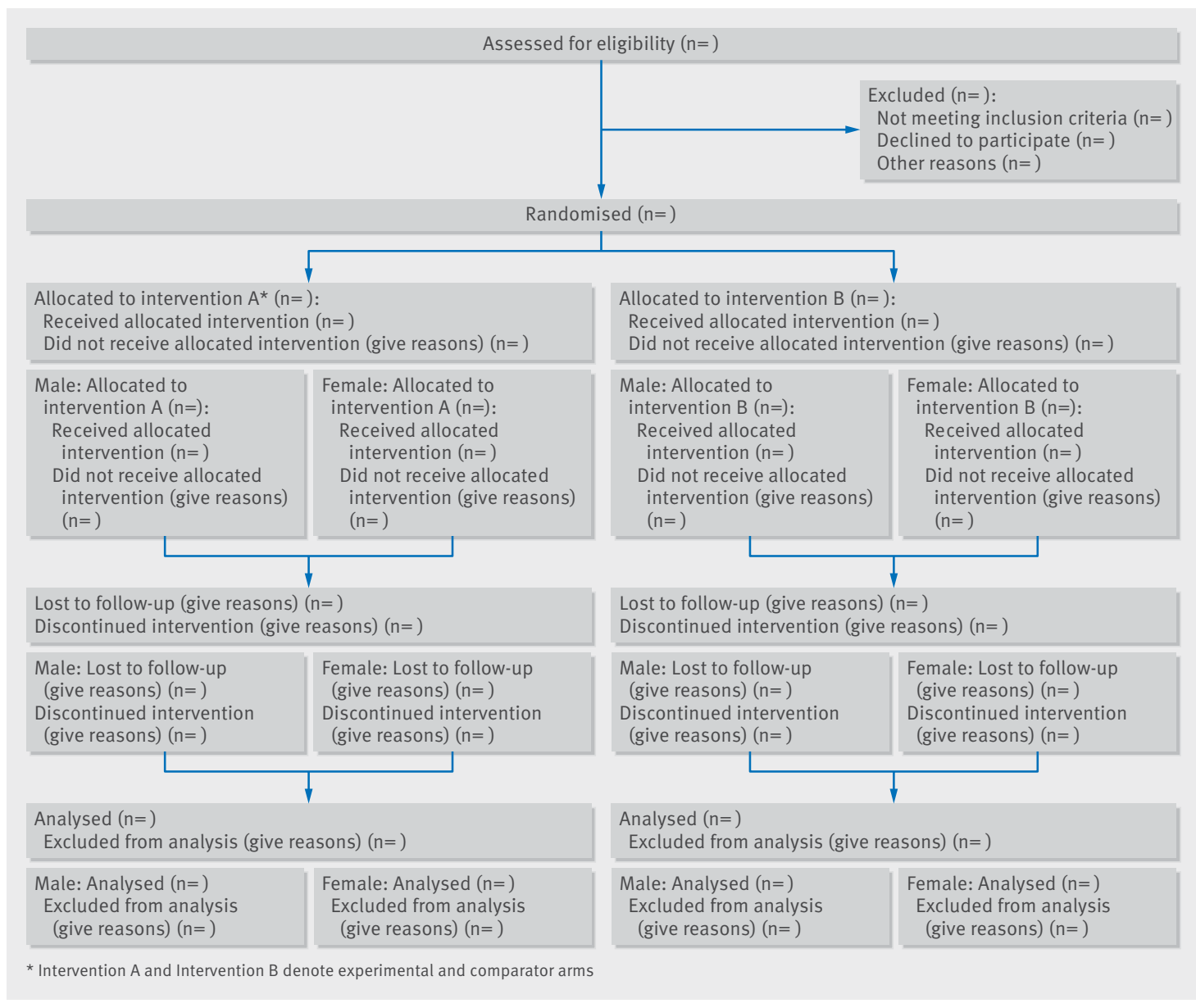

Fig 2 | CONSORT flow diagram

reported, allowing readers to construct alternative equity metrics. If the paper reports effects on equity in terms of absolute differences in effects, information about the baseline values of outcomes will allow readers to reframe the findings in terms of relative or proportional differences in effects compared with baseline.

There are no extensions for the CONSORT items 16 and 17.

Item 18a

Standard CONSORT item-Results of any other analyses performed, including subgroup analyses and adjusted analyses, distinguishing pre-specified from exploratory.

Extension for health equity relevant trials-Give the results of additional analytic approaches related to equity objectives distinguishing prespecified from exploratory.

Example-"In the group of patients without postsecondary education $(\mathrm{n}=90)$ the composite score showed a significant improvement in favour of the person centred care intervention $(\mathrm{n}=40)$ versus usual care $(\mathrm{n}=50)$ at 6 months $(35.0 \%, \mathrm{n}=14 \mathrm{~V} 16.0 \%$, $\mathrm{n}=8 ; \mathrm{OR}=2.8,95 \% \mathrm{CI}: 1.0$ to $7.7 ; \mathrm{P}=0.041)$. Among patients with postsecondary education $(n=109)$, a difference (although non-significant) in favour of the
PCC intervention $(n=54)$ versus usual care $(n=55)$ was detected in the composite score $(13.0 \%, n=7 \quad V$ $3.6 \%, \mathrm{n}=2$; $\mathrm{OR}=3.9,95 \% \mathrm{CI}: 0.8$ to $19.9 ; \mathrm{P}=0.097) \ldots$ because this analysis is post hoc, no firm conclusions can be drawn." 58

Explanation-As above, additional analytic approaches (including quantitative, qualitative, or graphical) may be needed to achieve equity objectives. These may be described in follow-up (secondary) publications of a randomised trial. If so, the authors should specify which of these analyses were stated a priori.

\section{Item $18 b$}

Standard CONSORT item-None.

Extension for health equity relevant trials-Details of implementation (coverage, intensity) in each trial arm across relevant PROGRESS-Plus characteristics.

Example-“Home visits were pro-poor, with more women in the poorest quintile visited by a CHW [community health worker] compared to families in the least poor quintile, and more women who delivered at home visited by a CHW after birth (73.6\%) compared to those who delivered in a hospital or health facility (59.7\%) $(\mathrm{P}<0.001)$. . 59

Explanation-We propose that authors assess and describe actual reach and implementation of the 
intervention (including coverage, intensity, uptake) across PROGRESS-Plus characteristics to understand how and the extent to which interventions work. ${ }^{60}$ The funnel of attrition may be very different for disadvantaged populations than more advantaged populations. ${ }^{13}$ Details on actual implementation are also important for applicability.

\section{Item 19}

Standard CONSORT item-All important harms or unintended effects in each group (for specific guidance see CONSORT for harms).

Extension for health equity relevant trials-Report whether inequities caused by the intervention (such as unintended effects) were assessed.

Example-A shared decision making intervention was found to be less effective for people with low socioeconomic status, ${ }^{61}$ which may inadvertently widen the gap between rich and poor, thus increasing inequities.

Explanation-Interventions may generate inequities if the most disadvantaged benefit less from the intervention or experience greater harms. ${ }^{62}$ If such interventions are incorporated into care, they could further widen the gap in health status across socioeconomic status, and decision makers may find this information relevant.

\section{Discussion}

Item 20

Standard CONSORT item-Trial limitations, addressing sources of potential bias, imprecision, and, if relevant, multiplicity of analyses.

Extension for health equity relevant trials-Report any limitations related to assessing effects on health equity.

Example-“The number of observations is relatively small; hence, the study may not have had enough power to identify real differences between the intervention and control groups. We, therefore, cannot rule out a possible equity impact of the intervention." 29

Explanation-Trials that aim to assess effects on health equity may not achieve sufficient sample size, may have problems of recruitment or retention, or may have baseline imbalance in characteristics of interest (such as socioeconomic status). Although these limitations are not specific to trials relevant to health equity, the implications of these limitations on the reliability of the trial for assessing effects on health equity should be reported.

\section{Item 21}

Standard CONSORT item-Generalisability (external validity, applicability) of the trial findings.

Extension for health equity relevant trials-In addition, report applicability related to population of interest across PROGRESS-Plus characteristics.

Example-“This study was carried out in a single site, and risk behaviours in the sample may not be characteristic of other Roma communities or other populations."63

Explanation-We propose that trials report applicability related to the disadvantaged groups under study as evidence in one disadvantaged population may not apply across other axes of disadvantage. In addition, reporting the rationale for these judgments (such as the use of a logic framework or proposed mechanisms) will assist in considering applicability in other settings or populations.

There are no extensions for the CONSORT items 22-25.

\section{Discussion}

\section{Strengths and limitations of our approach}

We used a structured process to collect evidence about the need for these reporting guidelines, including empiric studies, key informant interviews, and an online survey, following accepted methods for developing reporting guidelines. ${ }^{27}$ One limitation of our approach is that we received only 168 responses to our online survey (approximately 20\% response rate). We think this is unlikely to seriously affect the generalisability of our results or the selection of items, as we found similar responses across different respondent types (for example, patients versus others or people from low and middle income countries versus others), and we triangulated the results with our key informant interviews and consensus panel, which also included representation across knowledge user types and methodologists.

\section{Postpublication activities, implementation, and journal endorsement}

Implementation of the CONSORT-Equity 2017 reporting guideline may require tailored messages and tools for specific audiences, such as journal editors, peer reviewers, patients, researchers, and funders. We will develop tools for specific audiences, including an abbreviated checklist, a peer reviewer too, and a checklist to guide design and development of a health equity relevant trial. We will disseminate the reporting guideline and checklist as well as audience specific tools in discipline specific forums (including journals, conferences, and newsletters).

We registered CONSORT-Equity 2017 with the library of reporting guidelines maintained by the Enhancing the Quality and Transparency of Health Research (EQUATOR) Network (www.equator-network. org) and published a protocol to promote awareness. ${ }^{14}$ Journal endorsement of CONSORT guidelines has been associated with better reporting of some items. ${ }^{26}$ The standard CONSORT guideline is endorsed by 585 journals (www.consort-statement.org/about-consort/ endorsers). We aim to contact journal editors to promote the use of the CONSORT-Equity extension by suggesting "standard" wording that could be included in instructions to authors; for example:

"[This journal] requires a completed CONSORTEquity 2017 checklist for randomised trials that are relevant to health equity. Reporting these minimum 
requirements will improve your manuscript and may enhance its chances for eventual publication."

Such wording will help send a clear message from journals to prospective authors and help avoid potential confusion. ${ }^{26}$ In addition, we will include CONSORT-Equity 2017 on the CONSORT website and explore how to include CONSORT-Equity in WebCONSORT, which is an online tool designed to facilitate reporting of trials. ${ }^{64}$

Evaluation of the impact of endorsement and implementation activities for reporting guidelines is uncommon (less than $10 \%$ of guidelines), so there is insufficient evidence to guide better implementation of these guidelines. ${ }^{65}$ We will assess impact of dissemination activities by citation rates in journal articles as well as mention of the checklist in the EQUATOR Network library, in instructions for authors in journals, or instructions for applicants in, for example, funding calls. We will seek partnerships with journals to prospectively evaluate the impact on reporting of health equity relevant trials using controlled studies.

\section{Conclusions}

All randomised trials contribute to an evidence base that will be applied at the population level. However, only some trials may be equity relevant. Equity relevant trials include those that are aimed specifically at people who are disadvantaged or those that collect data on impact for different socio-demographic groups. This work serves as an explicit prompt to carefully consider health equity issues in these health equity relevant trials and report these considerations in publications.

The CONSORT-Equity 2017 guidelines aim to improve the evidence base about the effects on health equity of health technologies, programmes, and policies. To encourage widespread uptake of the guideline, we used an inclusive approach to engage planned users in the development of the research. We followed a structured approach to developing these guidelines, including systematic reviews and methodology surveys, broad user engagement to seek opinions, and key informant interviews. Providing the results of these background studies were a strength of the process; details of which will be published subsequently in a series of methodological papers.

We hope that CONSORT-Equity 2017 leads to better reporting of health equity issues in randomised trials, and eventually a strengthened evidence base for promoting health equity. This will not only improve fairness in promoting and protecting health but will in many contexts enhance the overall impact of interventions.

CONSORT-Equity and Boston Equity Symposium participants: Rebecca Armstrong, Yukiko Asada, Kristine Bærøe, Sarah Baird, Zulfiqar A Bhutta, Yvonne Boyer, Luis Gabriel Cuervo, Sarah JL Edwards, David Gough, Regina Greer-Smith, Jeremy M Grimshaw, Kjell Arne Johansson, Caroline Nyamai Kisia, Elizabeth Kristjansson, Elizabeth Loder, Anne Lyddiatt, Lawrence Mbuagbaw, Jessie McGowan, David Moher, Tomas Pantoja, Jennifer Petkovic, Mark Petticrew, Kevin Pottie, Bjarne Robberstad, Beverley Shea, Monica Taljaard, Stéphane Verguet, Jimmy Volmink, Charles Weijer, George A Wells, Gry Wester, Howard White, Margaret Whitehead, Manosila Yoganathan
The authors gratefully acknowledge the contributions of Heather Swan, Shalini Suresh, Elizabeth Ghogomu, William McLeod, Christian Charbonneau, Anna Malinowski, Sean Lim, Nabeelah Ahmed, Laura Silva Nossa, Christine Smith, Maria Helena Jacob, Jessica Trawin, and Victoria Frost in assisting with collecting data and examples of health equity relevant trials

Contributors and sources: VW and PT conceptualised the study design. MY, JJ, VW, PT, MT, LM, and GAW collected data, and all authors provided input on the direction of the study and content of the checklist. All authors approved the final version of the manuscript. After completing the PRISMA-Equity 2012 reporting guideline for equity focused systematic reviews, VW and PT sought funding in 2014 to develop a parallel extension for CONSORT for health equity relevant trials, because one of the main limitations of conducting an equity focused review is the lack of detail in primary randomised trials. VW and PT invited international colleagues with a diversity of academic and knowledge user perspectives to join the research team. We invited J] to lead the development of our integrated knowledge translation strategy, including developing an advisory board. We joined forces with ON and HS in April 2016 because they contacted us to join a very similar initiative, with funding from CISMAC, aimed at improving the reporting of health equity in randomised trials relating to maternal and child health. VW is the guarantor for the article.

Funding: This work was funded by a CIHR operating grant (REF \#-MOP 133 556), the Research Council of Norway through its Centres of Excellence scheme and the University of Bergen (UiB), Norway to the Centre for Intervention Science in Maternal and Child Health (CISMAC; project number 223269) and an Ontario Early Researcher Award held by VW. The funders had no role in the study design, the collection, analysis, and interpretation of data or the writing of the article or the decision to submit it for publication.

Competing interests: All authors have completed the Unified Competing Interest form (available on request from the corresponding author) and declare: that VW reports a grant from the Canadian Institutes of Health Research and a grant from the Ontario Early Researcher Award during the conduct of the study; RC reports grants from NIHR during the conduct of the study, and LC reports that his employer is the Pan American Health Organisation, which is part of the World Health Organization, an intergovernmental organisation that is part of the UN system. Tackling health equity issues is in the mission and vision of the organisation. His salary is paid from the organisation's budget, which is approved and funded by PAHO and WHO member states. His work includes monitoring and supporting the advancement of the Policy on Research for Health CD49/10 that specifically calls for the development of methods and better reporting of equity issues in research. The funders had no role in the study design. There were no other relationships or activities that could appear to have influenced the submitted work.

Transparency statement: VW affirms that the manuscript is an honest, accurate, and transparent account of the study being reported; that no important aspects of the study have been omitted; and that any discrepancies from the study as planned (and, if relevant, registered) have been explained.

Protocol: Welch V, Jull J, Petkovic J, et al. Protocol for the development of a CONSORT-equity guideline to improve reporting of health equity in randomised trials. Implement Sci. 2015 Oct 21;10:146. doi: 10.1186/s13012-015-0332-z.

Checklist for study type: No appropriate checklist is available for reporting guidelines. We followed the methods of Moher et al 2010.

Data sharing statement: Background material for the consensus meeting are available upon request from the first author, Vivian Welch at vivian.welch@uottawa.ca

1 Chetty R, Stepner M, Abraham S. The association between income and life expectancy in the United States, 2001-2014. JAMA 2016;315:1750-66. doi:10.1001/jama.2016.4226.

2 Marmot M, Friel S, Bell R, Houweling TA, Taylor S. Commission on Social Determinants of Health. Closing the gap in a generation: health equity through action on the social determinants of health. Lancet 2008;372:1661-9. doi:10.1016/S01406736(08)61690-6.

3 Marmot M, Allen J, Bell R, Goldblatt P. Building of the global movement for health equity: from Santiago to Rio and beyond Lancet 2012;379:181-8. doi:10.1016/S0140-6736(11)61506-7.

4 World Health Organization. World Health Organization handbook for guideline development. Chapter 5: Incorporating equity, gender, human rights and social determinants into guideline development. 2014 
5 NICE. Positively equal: A guide to addressing equality issues in developing NICE clinical guidelines. 2012. https://www.nice.org.uk/ media/default/about/what-we-do/nice-guidance/nice-guidelines/ clinical-guidelines/positively-equal.pdf

6 Welch V. GRADE Equity series: Why health equity and guidelines?J Clin Epidemiol [forthcoming].

7 Pan American Health Organization and World Health Organization. Policy on research for health. Document CD49/10 and resolution CD49.R10. Washington, DC. 49th Directing Council and 61st Session of the Regional Committee of WHO 2009.http://www.paho.org/ researchportal/policy.

8 63rd World Health Assembly. WHO strategy on research for health; WHO roles and responsibilities on health research: document WHA63.22 and Resolution. Geneva. 2010.http://apps.who.int/ gb/e/e_wha63.htm

9 Boruch RF. Randomized experiments for planning and evaluation: A practical guide, vol. 44. Sage 1997.

10 Zwarenstein M, Oxman A. Pragmatic Trials in Health Care Systems (PRACTIHC). Why are so few randomized trials useful, and what can we do about it?] Clin Epidemiol 2006;59:1125-6. doi:10.1016/j. jclinepi.2006.05.010

11 Grimshaw IM, Presseau J, Tetroe J. Looking inside the black box: results of a theory-based process evaluation exploring the results of a randomized controlled trial of printed educational messages to increase primary care physicians' diabetic retinopathy referrals [Trial registration number ISRCTN72772651][Trial registration number ISRCTN72772651]. Implement Sci 2014;9:86. doi:10.1186/17485908-9-86

12 Macintyre S. Good intentions and received wisdom are not good enough: the need for controlled trials in public health. $)$ Epidemiol Community Health 2011;65:564-7. doi:10.1136/ jech.2010.124198.

13 White $\mathrm{H}$. Using the causal chain to make sense of the numbers 2013. http://www.3ieimpact.org/en/announcements/2013/02/12 using-causal-chain-make-sense-numbers/

14 Welch V, Jull J, Petkovic J. Protocol for the development of a CONSORTequity guideline to improve reporting of health equity in randomized trials. Implement Sci 2015;10:146. doi:10.1186/s13012-015 0332-z.

15 King G, Gakidou E, Imai K. Public policy for the poor? A randomised assessment of the Mexican universal health insurance programme. Lancet 2009;373:1447-54. doi:10.1016/S0140-6736(09) 60239-7.

16 O'Neill J, Tabish H, Welch V. Applying an equity lens to interventions: using PROGRESS ensures consideration of socially stratifying factors to illuminate inequities in health. J Clin Epidemiol 2014;67:56-64. doi:10.1016/j.jclinepi.2013.08.005.

17 Oliver SKJ, Caird J. Health promotion, inequalities and young people's health. A systematic review of research. EPPI-Centre, Social Science Research Unit, Institute of Education, University of London, 2008, http://eppi.ioe.ac.uk/cms/Default.aspx?tabid=2410\&language= en-US.

18 Furler J, Magin P, Pirotta M, van Driel M. Participant demographics reported in "Table 1 " of randomised controlled trials: a case of "inverse evidence"? Int J Equity Health 2012;11:14 doi:10.1186/1475-9276-11-14

19 Welch V, Petticrew M, Ueffing E. Does consideration and assessment of effects on health equity affect the conclusions of systematic reviews? A methodology study. PLoS One 2012;7:e31360. doi:10.1371/journal.pone.0031360.

20 Godden S, Ambler G, Pollock AM. Recruitment of minority ethnic groups into clinical cancer research trials to assess adherence to the principles of the Department of Health Research Governance Framework: national sources of data and general issues arising from a study in one hospital trust in England. J Med Ethics 2010;36:358 62. doi:10.1136/ime.2009.033845.

21 Kasenda B, Schandelmaier S, Sun X. DISCO Study Group. Subgroup analyses in randomised controlled trials: cohort study on trial protocols and journal publications. BMJ 2014;349:g4539. doi:10.1136/bmi.g4539

22 Heidari S, Babor TF, De Castro P, Tort S, Curno M. Sex and gender equity in research: rationale for the SAGER guidelines and recommended use. Res Integr Peer Rev. 2016;1:2. doi:10.1186/ s41073-016-0007-6.

23 Beard JR, Officer A, de Carvalho IA. The world report on ageing and health: a policy framework for healthy ageing Lancet 2016;387:2145-54. doi:10.1016/S0140-6736(15) 00516-4

24 Petkovic J, Epstein J, Buchbinder R. Toward ensuring health equity: readability and cultural equivalence of OMERACT patient-reported outcome measures. J Rheumatol 2015;42:2448-59. doi:10.3899/ jrheum.141168.

25 Mbuagbaw L, Aves T, Shea B. Considerations and guidance in designing equity-relevant clinical trials. Int J Equity Health 2017;16:93. doi:10.1186/s12939-017-0591-1.
26 Turner L, Shamseer L, Altman DG, Schulz KF, Moher D. Does use of the CONSORT Statement impact the completeness of reporting of randomised controlled trials published in medical journals? A Cochrane review. Syst Rev 2012;1:60. doi:10.1186/2046-4053-1-60

27 Moher D, Schulz KF, Simera I, Altman DG. Guidance for developers of health research reporting guidelines. PLoS Med 2010;7:e1000217. doi:10.1371/journal.pmed.1000217.

28 Canadian Institute of Health Research (CIHR). Guide to knowledge translation planning at cihr: integrated and end-of-grant approaches. 2015. http://www.cihr-irsc.gc.ca/e/45321.html

29 Eide KT, Fadnes LT, Engebretsen IM. Impact of a peer-counseling intervention on breastfeeding practices in different socioeconomic strata: results from the equity analysis of the PROMISE-EBF trial in Uganda. Glob Health Action 2016;9:30578. doi:10.3402/gha. v9.30578.

30 Saleem AF, Mahmud S, Baig-Ansari N, Zaidi AKM. Impact of maternal education about complementary feeding on their infants' nutritional outcomes in low- and middle-income households: a communitybased randomized interventional study in Karachi, Pakistan. J Health Popul Nutr 2014;32:623-33.

31 Brown AF, Vassar SD, Connor KI, Vickrey BG. Collaborative care management reduces disparities in dementia care quality for caregivers with less education. J Am Geriatr Soc 2013;61:243-51. doi:10.1111/jgs.12079

32 Gupta S, Miller S, Koch M. Financial incentives for promoting colorectal cancer screening: a randomized, comparative effectiveness trial. Am J Gastroenterol 2016;111:1630-6. doi:10.1038/ ajg.2016.286

33 Surratt HL, O'Grady C, Kurtz SP, Levi-Minzi MA Chen M. Outcomes of a behavioral intervention to reduce HIV risk among drug-involved female sex workers. AIDS Behav 2014;18:726-39. doi:10.1007/ s10461-014-0723-1.

34 Foley L, Jiang Y, Ni Mhurchu C. The effect of active video games by ethnicity, sex and fitness: subgroup analysis from a randomised controlled trial. Int J Behav Nutr Phys Act 2014;11:46. doi:10.1186/1479-5868-11-46.

35 Kaufman CE, Whitesell NR, Keane EM. Effectiveness of circle of life, an HIV-preventive intervention for American Indian middle school youths: a group randomized trial in a Northern Plains tribe. Am J Public Health 2014;104:e106-12. doi:10.2105/AJPH.2013.301822.

36 Rodrigue JR, Paek MJ, Egbuna O. Making house calls increases living donor inquiries and evaluations for blacks on the kidney transplant waiting list. Transplantation 2014;98:979-86. doi:10.1097/ TP.0000000000000165

37 Raj A, Ghule M, Ritter J. Cluster randomized controlled trial evaluation of a gender equity and family planning intervention for married men and couples in rural India. PLoS One 2016;11:e0153190. doi:10.1371/journal.pone.0153190.

38 Krezanoski PJ, Comfort AB, Hamer DH. Effect of incentives on insecticide-treated bed net use in sub-Saharan Africa: a cluster randomized trial in Madagascar. Malar J 2010;9:186-186. doi:10.1186/1475-2875-9-186.

39 Hosseinipour MC, Bisson GP, Miyahara S. Adult AIDS Clinical Trials Group A5274 (REMEMBER) Study Team. Empirical tuberculosis therapy versus isoniazid in adult outpatients with advanced HIV initiating antiretroviral therapy (REMEMBER): a multicountry openlabel randomised controlled trial. Lancet 2016;387:1198-209. doi:10.1016/S0140-6736(16)00546-8

40 Wendler D, Emanuel EJ, Lie RK. The standard of care debate: can research in developing countries be both ethical and responsive to those countries' health needs?Am J Public Health 2004;94:923-8. doi:10.2105/AJPH.94.6.923

41 Lie RK, Emanuel E, Grady C, Wendler D. The standard of care debate: the Declaration of Helsinki versus the international consensus opinion. J Med Ethics 2004;30:190-3. doi:10.1136/ ime.2003.006031

42 Shaffer N, Chuachoowong R, Mock PA. Bangkok Collaborative Perinatal HIV Transmission Study Group. Short-course zidovudine for perinatal HIV-1 transmission in Bangkok, Thailand: a randomised controlled trial Lancet 1999:353.773-80 doi:10.1016/S01406736(98)10411-7

43 Obsuth I, Cope A, Sutherland A, Pilbeam L, Murray AL, Eisner M. London education and inclusion project (LEIP): exploring negative and null effects of a cluster-randomised school-intervention to reduce school exclusion-findings from protocol-based subgroup analyses. PLOS One 2016;11:e0152423. doi:10.1371/journal.pone.0152423.

44 Baird SJ, Garfein RS, McIntosh CT, Özler B. Effect of a cash transfer programme for schooling on prevalence of HIV and herpes simplex type 2 in Malawi: a cluster randomised trial. Lancet 2012;379:1320 9. doi:10.1016/S0140-6736(11)61709-1.

45 Forster A, Dickerson J, Young J. TRACS Trial Collaboration. A cluster randomised controlled trial and economic evaluation of a structured training programme for caregivers of inpatients after stroke: the TRACS trial. Health Technol Assess 2013;17:1-216. doi:10.3310/ hta17460. 
46 Sukala WR, Page R, Lonsdale C. Exercise improves quality of life in indigenous Polynesian peoples with type 2 diabetes and visceral obesity. J Phys Act Health 2013;10:699-707. doi:10.1123/ jpah.10.5.699

47 Raine R, Moss SM, von Wagner C. A national cluster-randomised controlled trial to examine the effect of enhanced reminders on the socioeconomic gradient in uptake in bowel cancer screening. $\mathrm{Br}$ J Cancer 2016;115:1479-86. doi:10.1038/bjc.2016.365.

48 Chen JT, Beckfield J, Waterman PD, Krieger N. Can changes in the distributions of and associations between education and income bias temporal comparisons of health disparities? An exploration with causal graphs and simulations. Am J Epidemiol 2013;177:870-81. doi:10.1093/aje/kwt041.

49 Schulz KF, Altman DG, Moher D. CONSORT Group. CONSORT 2010 statement: updated guidelines for reporting parallel group randomized trials. Obstet Gynecol 2010;115:1063-70. doi:10.1097/AOG.0b013e3181d9d421.

50 Council for International Organizations of Medical Sciences (CIOMS). International ethical guidelines for health-related research involving humans. http://cioms.ch/shop/product/international-ethicalguidelines-for-health-related-research-involving-humans/. 2016: 122

51 Canadian Institute of Health Research; Natural Sciences and Engineering Research Council of Canada; Social Sciences and Humanities Research Council of Canada. Tri-council policy statement: ethical conduct for research involving humans (TCPS2). 2014. http:// www.pre.ethics.gc.ca/pdf/eng/tcps2-2014/TCPS 2 FINAL Web.pdf

52 World Medical Association. World Medical Association Declaration of Helsinki: ethical principles for medical research involving human subjects. JAMA 2013;310:2191-4. doi:10.1001/ jama.2013.281053.

53 National Commission for the Protection of Human Subjects of Biomedical and Behavioral Research. The Belmont report: ethical principles and guidelines for the protection of human subjects of research. https://www.hhs.gov/ohrp/regulations-and-policy/ belmont-report/index.html\#. 1979.

54 Weijer C, Grimshaw IM, Eccles MP. Ottawa Ethics of Cluster Randomized Trials Consensus Group. The Ottawa statement on the ethical design and conduct of cluster randomized trials. PLOS Med 2012;9:e1001346. doi:10.1371/journal.pmed.1001346.

55 Pilcher J, Patel M, Smith A. SMART Study Group. Combination budesonide/formoterol inhaler as maintenance and reliever therapy in Māori with asthma. Respirology 2014;19:842-51. doi:10.1111/ resp.12319.

56 Booth AR, Norman P, Goyder E, Harris PR, Campbell MJ. Pilot study of a brief intervention based on the theory of planned behaviour and self-identity to increase chlamydia testing among young people living in deprived areas. Br J Health Psychol 2014;19:636-51. doi:10.1111/bjhp.12065

57 King AC, Cao D, Zhang L, O'Malley SS. Naltrexone reduction of long-term smoking cessation weight gain in women but not men: a randomized controlled trial. Biol Psychiatry 2013;73:924-30 doi:10.1016/j.biopsych.2012.09.025.
58 Fors A, Gyllensten H, Swedberg K, Ekman I. Effectiveness of person-centred care after acute coronary syndrome in relation to educational level: Subgroup analysis of a two-armed randomised controlled trial. Int J Cardiol 2016;221:957-62. doi:10.1016/j. ijcard.2016.07.060.

59 Waiswa P, Pariyo G, Kallander K. Effect of the Uganda Newborn Study on care-seeking and care practices: a cluster-randomised controlled trial. Glob Health Action 2015;8:24584. doi:10.3402/ gha.v8.24584

60 Craig P, Dieppe P, Macintyre S, Michie S, Nazareth I, Petticrew M. Medical Research Council Guidance. Developing and evaluating complex interventions: the new Medical Research Council guidance. BM/2008:337:a1655. doi:10.1136/bmj.a1655.

61 Marteau TM, Mann E, Prevost AT. Impact of an informed choice invitation on uptake of screening for diabetes in primary care (DICISION): randomised trial. BMJ 2010;340:C2138. doi:10.1136/ bmj.c2138

62 Lorenc T, Petticrew M, Welch V, Tugwell P. What types of interventions generate inequalities? Evidence from systematic reviews. J Epidemiol Community Health 2013;67:190-3. doi:10.1136/jech-2012201257.

63 Kelly JA, Amirkhanian YA, Kabakchieva E. Prevention of HIV and sexually transmitted diseases in high risk social networks of young Roma (Gypsy) men in Bulgaria: randomised controlled trial. BMJ 2006;333:1098-1098. doi:10.1136/bmj.38992.478299.55.

64 Hopewell S, Boutron I, Altman DG. Impact of a web-based tool (WebCONSORT) to improve the reporting of randomised trials: results of a randomised controlled trial. BMC Med 2016;14:199. doi:10.1186/s12916-016-0736-x.

65 Stevens A, Shamseer L, Weinstein E. Relation of completeness of reporting of health research to journals' endorsement of reporting guidelines: systematic review. BMJ 2014;348:g3804. doi:10.1136/ bmj.g3804

Web appendix 1: Distribution of online survey Web appendix 2: Guidance and policy statements reviewed as background material

Web table 1: Survey participants' description Web table 2: Survey responses for CONSORT-Equity extension items

Web table 3: Boston Equity Symposium participants' background

Web table 4: Example of reporting baseline characteristics across sociodemographic characteristics, relevant for health equity 